\title{
Warped molecular gas disk in NGC 3718
}

\author{
J.-U. Pott ${ }^{1}$, M. Hartwich ${ }^{1}$, A. Eckart ${ }^{1}$, S. Leon ${ }^{2}$, M. Krips ${ }^{1,3}$, and C. Straubmeier ${ }^{1}$ \\ 1 Universität zu Köln, I. Physikalisches Institut, Zülpicherstrasse 77, 50937 Köln, Germany \\ 2 Instituto de Astrofísica de Andalucía (IAA), c/ Camino Bajo de Huétor 24, 18008 Granada, Spain \\ 3 Institut de Radio-Astronomie Millimétrique (IRAM), 300 rue de la Piscine, Domaine Universitaire, \\ 38406 Saint Martin d'Hères, France
}

Received 22 April 2003 / Accepted 1 October 2003

\begin{abstract}
We present the first observations of the $\mathrm{CO}(1-0), \mathrm{CO}(2-1)$ and $\mathrm{HCN}(1-0)$ rotational line emission of NGC 3718 , conducted with the IRAM $30 \mathrm{~m}$ telescope. The results of the data analysis show a thin strongly warped molecular gas disk harboring the active galactic nucleus (AGN). The ratio of the total molecular gas mass $\left(2 \times 10^{8} M_{\odot}\right)$ to the dynamical mass, enclosed within the same region, is found to be rather low $(\sim 0.17 \%)$, but still typical for spiral galaxies. The found molecular gas disk is well associated with the dust lane, visible at optical wavelengths. We traced the warped CO structure down to the central $20^{\prime \prime}$. In its outer region the molecular disk is well correlated with the HI distribution. The CO data is used to improve the kinematic modelling in the inner part of the galaxy $\left(10^{\prime \prime} \leq r \leq 120^{\prime \prime}\right)$ employing a tilted ring-model. Furthermore the properties of NGC 3718 are compared with those of its northern sky "twin" NGC 5128 (Centaurus A).
\end{abstract}

Key words. galaxies: individual: NGC 3718 - galaxies: active - galaxies: kinematics and dynamics - ISM: molecules

\section{Introduction}

The galaxy NGC 3718 (Arp 214) and its companion NGC 3729 form a galaxy pair (see Fig. 1) at a distance of about $13 \mathrm{Mpc}$ corresponding to a linear scale of $64 \mathrm{pc} / \operatorname{arcsec}^{1}$. They belong to the poorly defined Ursa Major cluster of galaxies, which shows a velocity dispersion of less than $150 \mathrm{~km} \mathrm{~s}^{-1}$ (Tully et al. 1996). In addition Garcia 1993 classified NGC 3718 and NGC 3729 as members of Group 241 in the LGG (Lyon Group of Galaxies) catalog. A low velocity dispersion indicates, that cannibalism of small galaxies is quite likely. The basic parameters of NGC 3718 and NGC 3729 are given in Table 1. The galaxies are also contained in the Hubble Atlas of Galaxies (Sandage 1961) and the Atlas of Peculiar Galaxies (Arp 1966), where an extremely dark dust feature and a relatively small nucleus of NGC 3718 become apparent (Arp 1973).

The most prominent optical feature of NGC 3718 is its large, warped dust lane running across the entire stellar bulge of the galaxy (see Fig. 2). A fine dust lane indicates high inclination of the galaxy with respect to the observer. While close to the center the dust is confined to a narrow lane showing a width of less than $2^{\prime \prime}$, at larger radii $\left(r \geq 15^{\prime \prime}\right)$ several smooth filaments across the bulge of the galaxy are visible. At a

Send offprint requests to: J.-U. Pott, e-mail: pott@ph1.uni-koeln.de

1 Throughout this work we assume $H_{0}=75 \mathrm{~km} \mathrm{~s}^{-1} \mathrm{Mpc}^{-1}$. The distance was calculated via the redshift $z=0.0033$ of NGC 3718 (NASA Extragalactic Database NED). Pierce \& Tully (1988) estimated a slightly differing distance of $(15.5 \pm 1.2) \mathrm{Mpc}$.

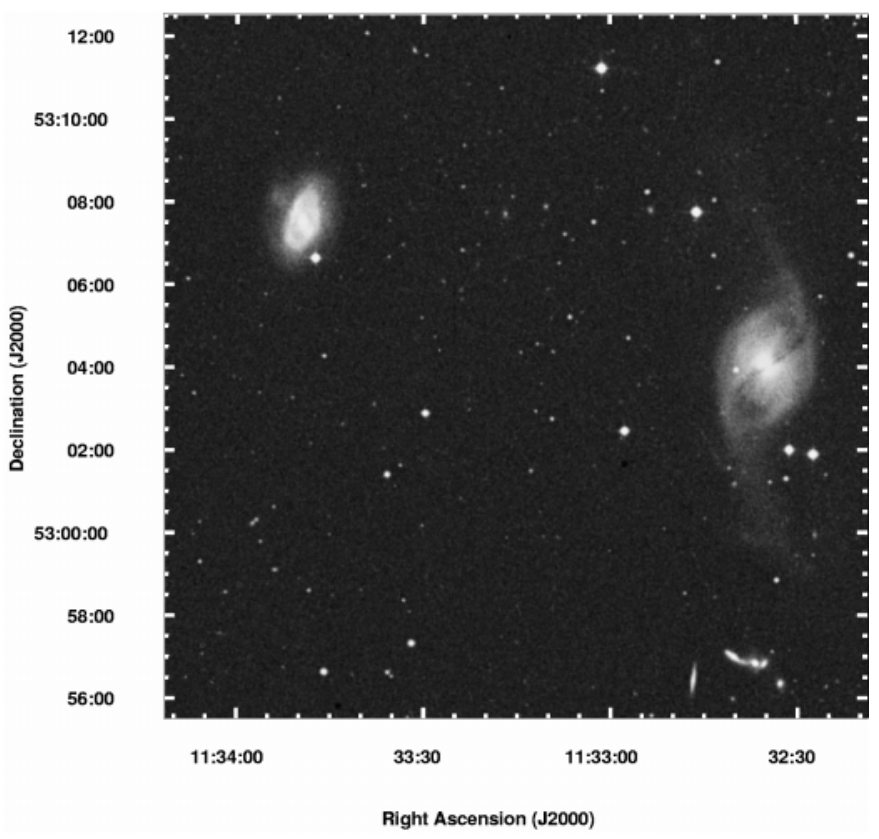

Fig. 1. Optical image of the pair NGC 3718 (to the west) and NGC 3729 (to the east). Taken from the DSS service.

separation of about 1.5 arcmin from the nucleus these dust filaments bend by almost $90^{\circ}$ towards the north and the south and remain visible over a distance of more than 6 arcmins $(24 \mathrm{kpc})$. Inspection of the dust lane shows that the warp signature goes all the way into the center of the galaxy. The optical appearance 
Table 1. Global parameters of NGC 3718 and NGC 3729.

\begin{tabular}{|c|c|c|c|}
\hline & NGC 3718 & NGC 3729 & references \\
\hline RA (J2000.0) & $11^{\mathrm{h}} 32^{\mathrm{m}} 35^{\mathrm{s}} \cdot 3$ & $11^{\mathrm{h}} 33^{\mathrm{m}} 49^{\mathrm{s}} .4$ & $\mathrm{NED}^{a}$ \\
\hline $\operatorname{Dec}(\mathrm{J} 2000.0)$ & $+53^{\circ} 04^{\prime} 01^{\prime \prime}$ & $+53^{\circ} 07^{\prime} 32^{\prime \prime}$ & $\mathrm{NED}^{a}$ \\
\hline Host type $\mathrm{e}^{b}$ & $\mathrm{Sa}$ & SBab & Verheijen \& Sancisi (2001) \\
\hline Nucleus type ${ }^{c}$ & L 1.9 & $\mathrm{H}$ & Ho et al. (1997) \\
\hline$B$-magnitude app./abs. (mag) & $11.59 /-19.01$ & $12.03 /-18.57$ & $\mathrm{NED}^{a}$ \\
\hline$R_{80}^{B \quad d}$ & $220^{\prime \prime}$ & $60^{\prime \prime}$ & Tully et al. (1996) \\
\hline Radio flux density S [mJy] at $10.7 \mathrm{GHz}$ & $8.5^{e}$ & $6^{f}$ & ${ }^{e}$ Nagar et al. (2001), ${ }^{f}$ Niklas et al. (1995) \\
\hline Radio flux density S [mJy] at $1.49 \mathrm{GHz}^{g}$ & 18.3 & 20.5 & Condon (1987) \\
\hline Radio flux density S [mJy] at $1.4 \mathrm{GHz}^{h}$ & $<50$ & $<50$ & Hummel (1980) \\
\hline Atomic gas mass $M_{\mathrm{H}}$ & $6 \times 10^{9} M_{\odot}$ & $0.15 \times 10^{9} M_{\odot}$ & Schwarz (1985) \\
\hline Total mass & $400 \times 10^{9} M_{\odot}$ & $40 \times 10^{9} M_{\odot}$ & Schwarz (1985) \\
\hline Position angle of major axis ${ }^{i}$ & $15^{\circ}$ & $-16^{\circ}$ & Verheijen \& Sancisi (2001) \\
\hline Inclination $^{i}$ & $68^{\circ}$ & $48^{\circ}$ & Verheijen \& Sancisi (2001) \\
\hline Heliocentric velocity ${ }^{i}$ & $993 \pm 1 \mathrm{~km} \mathrm{~s}^{-1}$ & $1060 \pm 1 \mathrm{~km} \mathrm{~s}^{-1}$ & Verheijen \& Sancisi (2001) \\
\hline
\end{tabular}

${ }^{a}$ NED: NASA/IPAC Extragalactic Database.

${ }^{b}$ Morphological classification. S: Spiral; SB: Barred Spiral.

${ }^{c}$ Spectroscopic classification. L1.9: LINER; H: HII nucleus.

${ }^{d}$ Radius containing $80 \%$ of the radiation in the $B$-band.

${ }^{e}$ Calculated with a spectral index of $\alpha=1.05$ based on flux densities at $8.4 \mathrm{GHz}$ and $15 \mathrm{GHz}$.

${ }^{g}$ Integrated VLA continuum flux density.

${ }^{h}$ Total WSRT continuum flux density.

${ }^{i}$ From HI with PA pointing to the approaching major semi-axis.

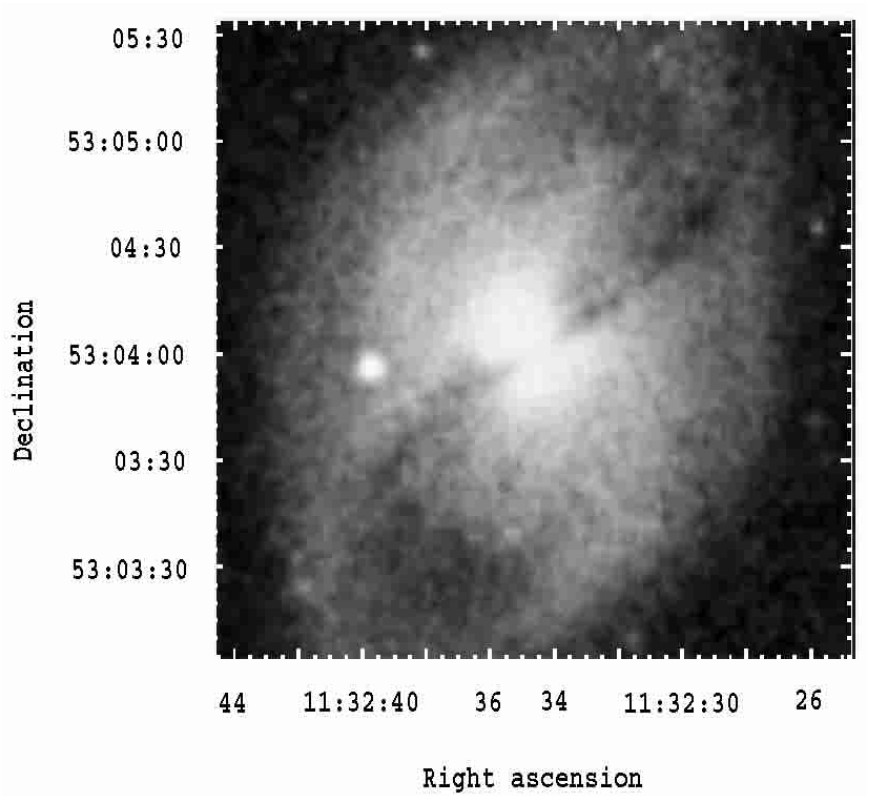

Fig. 2. Optical image of NGC 3718. Taken from the DSS survey.

of NGC 3718 (see Fig. 2) gave reason to different classifications. It was first listed as S0p by Holmberg (1958) due to the shape of its stellar bulge. Later it was classified as SB(s)ap by de Vaucouleurs et al. (1991) mainly due to the mentioned dust feature which was thought to be indicative of a bar.

The warped structure, indicated by the optical observations, is also present in the atomic gas (Schwarz 1985). The uniqueness of the $\mathrm{HI}$ distribution is that its inner and outer gas orbits are oriented nearly perpendicular with respect to each other. This led to the fact that NGC 3718 is often classified as a polar-ring galaxy (e.g. Reshetnikov \& Combes 1994).
The butterfly-like structure in Fig. 2 resembles the optical appearance of Centaurus A (NGC 5128) (Dufour et al. 1979). Thus NGC 3718 can be regarded as the northern hemisphere counterpart of Centaurus A. Also the neutral gas kinematics in both galaxies are found to be similar. Beside the warp the HI maps of NGC 3718 (Schwarz 1985) show a depletion of HI gas towards the central arcminute right on the dust lane. This is the location, where - like in Cen A (Eckart et al. 1990a; Wild et al. 1997) - the molecular gas takes over and becomes the dominant component of the neutral (atomic and molecular) ISM. In Centaurus A a warping of the molecular gas disk in the inner $1 \mathrm{kpc}$ was invoked to explain its dust lane and the associated complex kinematics (Sparke 1996; Quillen et al. 1992; Quillen et al. 1993).

NGC 3718 contains an active nucleus. Ho et al. (1997) classified NGC 3718 as a LINER 1.9 galaxy (see Table 1). The nucleus shows weak, broad $\mathrm{H} \alpha$ emission with $F W H M=$ $2350 \mathrm{~km} \mathrm{~s}^{-1}$. Filippenko et al. (1985) report strong [O I] $\lambda 6300$ with $F W H M=570 \mathrm{~km} \mathrm{~s}^{-1}$ indicative of a hidden AGN (Ho et al. 1997). Burke \& Miley (1973) found a radio source at the position of the nucleus of NGC 3718, reporting a $1415 \mathrm{MHz}$ flux density of $(20 \pm 5) \mathrm{mJy}$. The radio emission is at the lower end of the range of Seyfert galaxies, but exceeds that of most normal galaxies. Using the HST, Barth et al. (1998) searched for ultraviolet emission in LINER galaxies and did not detect NGC 3718. A possible explanation could be that the UV sources are obscured by dust (Maoz et al. 1995), which is reasonable in the case of NGC 3718.

To understand the evolution of galaxies it is essential to study the dynamics of the molecular gas. Similar to bars, warps may be an important element of galaxy evolution. It is believed that the formation of bulges is coupled to gas relocation due to a barred potential, as described by Combes (1990). In addition 
molecular gas has to be moved from large radii (kpc-scale) to a few parsecs in order to fuel the processes related to the nuclear activity like accretion onto blackholes and nuclear starbursts. All questionable fueling mechanisms have to be able to explain the necessary removal of angular momentum of the infalling matter.

How gas may easily be transported towards the center of such disks has recently been shown by Duschl et al. (2000). The authors suggest that viscosity within such gaseous disks may provide an efficient AGN fueling mechanism. Other processes, which enable the gas infall into the central few parsec, deal with nuclear bars (Shlosman et al. 1989), lopsidedness or $m=1$ instabilities (García-Burillo et al. 2000) and nuclear spiral density waves (Englmaier \& Shlosman 2000). If only one of these mechanisms or a combination of them usually accounts for the gas infall, is still unknown.

To derive the intrinsic kinematical data from the observation, it is crucial to know if the gas disks are warped or not. Furthermore warped disks definitely have an influence on how well the nucleus is hidden and/or fed with gas. Our recent high angular $\left(\sim 0.7^{\prime \prime}\right)$ resolution interferometric observations of other nearby galaxies with active nuclei (NGC 3227: Schinnerer et al. 2000a; NCG 1068: Schinnerer et al. 2000b; Arp 220: Eckart \& Downes 2001) show that the CO gas can be warped down to radii of $\sim 100 \mathrm{pc}$. Furthermore observations of $\mathrm{H}_{2} \mathrm{O}$ masers near the central engine in two nearby Seyfert galaxies show that also the central 1 pc disks can be warped (NGC 1068: Greenhill \& Gwinn 1997; NGC 4258: Miyoshi et al. 1995). Thus in general warped gas disks have been found in nearby galaxies at all linear scales.

In the course of our ongoing study of the dynamics of active galaxies (García-Burillo et al. 2003a,b) NGC 3718 turns out to be a special case because of the large range of radii, over which the gas distribution appears to be warped. Its gas is warped on kpc scales possibly due to interaction with an outer galactic halo. In this article it is shown, that the warp continues toward $r \sim 500 \mathrm{pc}$. And the recently obtained interferometric data strongly suggests, that even at radii smaller than $500 \mathrm{pc}$ the molecular gas disk may be warped as well. The detailed analysis of these interferometric data of the molecular gas distribution will be presented in a forthcoming article (Krips et al. in prep.). Thus we suggest to use the here presented data in any (statistical) investigation, which aims on answering the questions, how small-scale warps are connected to the large-scale HI warps and what are the implications for the gas transport?

In the concrete case of NGC 3718 we can present for the first time a model of the molecular gas distribution down to $r \sim 500 \mathrm{pc}$ and therefore test (at these scales) the existance of a molecular bar, which might have been hidden within the stellar bulge and the dust lane.

In Sect. 2 we describe our observations of $\mathrm{CO}(1-0)$, $\mathrm{CO}(2-1)$ and $\mathrm{HCN}(1-0)$. Section 3 deals with the reduction and analysis of the data of NGC 3718. There we describe the molecular gas distribution (Sect. 3.1) as well as dynamical mass estimations (Sect. 3.2). Furthermore the $\mathrm{CO}(2-1)$ to $\mathrm{CO}(1-0)$ line intensity ratios (Sect. 3.3), the FIR-properties (Sect. 3.4) and conclusions about the dense molecular gas and the star formation are discussed (Sect. 3.5). In Sect. 4
Table 2. Parameters of the two observing runs in November 1999 and June 2001.

\begin{tabular}{llll}
\hline \hline & $\mathrm{CO}(1-0)$ & $\mathrm{CO}(2-1)$ & $\mathrm{HCN}(1-0)$ \\
\hline Sky frequency [GHz] & 114.88927 & 229.77414 & 88.337936 \\
Beam size [arcsec] & 21.0 & 10.5 & 27.5 \\
Forward efficiency & 0.9 & 0.84 & 0.9 \\
Beam efficieny & 0.73 & 0.52 & 0.77 \\
\hline
\end{tabular}

we present a detailed kinematical modelling of the molecular gas which explains the three dimensional gas distribution and the observed peculiarities. Then a comparison of properties of NGC 3718 with NGC 5128 is given (Sect. 5). Section 6 summarizes the results of the previous sections.

\section{Observations}

Observations were carried out in November 1999 and June 2001 using the IRAM 30 meter radiotelescope on Pico Veleta, near Granada, Spain. Single-sideband SIS receivers were tuned to the $\mathrm{CO}(1-0), \mathrm{CO}(2-1)$ and $\mathrm{HCN}(1-0)$ transitions redshifted to the velocity of NGC 3718, namely $994 \mathrm{~km} \mathrm{~s}^{-1}$. Weather conditions were excellent during the run with typical system temperatures of 200-300 K. For each line a $512 \times 1 \mathrm{MHz}$ channel filter bank was used with a velocity resolution smoothed to about $20 \mathrm{~km} \mathrm{~s}^{-1}$ in order to improve the signal to noise of the individual spectra. At $88 \mathrm{GHz}, 115 \mathrm{GHz}$ and $230 \mathrm{GHz}$ we assume a HPBW of $27.5^{\prime \prime}, 21^{\prime \prime}$ and $10.5^{\prime \prime}$, respectively. Frequent observations of continuum sources provided a pointing accuracy of $3^{\prime \prime}$ (Greve et al. 1996). The parameters for the two observing runs are summarized in Table 2 . We used the wobbler with a switch cycle of $4 \mathrm{~s}$ and a beam throw of 90 $240^{\prime \prime}$. At least 4 to 5 times per hour we performed a chopper wheel calibration on a load at ambient temperature and on a cold load $(77 \mathrm{~K})$. The line temperatures are expressed in the $T_{\mathrm{A}}^{*}$ scale, antenna temperature corrected for atmospheric attenuation and rear sidelobes. The beam efficiencies that were used at each frequency to convert the $T_{\mathrm{A}}^{*}$ scale into the main beam brightness temperature scale are given in Table 2. Baselines were flat and only linear polynomials were used for correction.

The first observations in November 1999 covered a region of approximately $200^{\prime \prime} \times 50^{\prime \prime}$ along the dust lane at a position angle of $-66^{\circ}$ extending symmetrically around the nucleus. The beginning of the warp of the gas disk at an offset of $\pm 60^{\prime \prime}$ from the center along the major axis was covered as well. $\mathrm{HCN}(1-0)$ was probed at 13 positions within the central 20". To improve the final $\mathrm{CO}(2-1)$ map (Fig. 6), the second observing run was conducted in June 2001, observing the central $80^{\prime \prime} \times 50^{\prime \prime}$. Further the integration time of the HCN(1-0) observation was increased this time to rise the SNR and get clearer detections. The pointing grid of both observations is shown in Fig. 3. The measurements were carried out using a beam separation of $14^{\prime \prime}$ (i.e. half the $88 \mathrm{GHz}$ beam size) over most of the area and $7^{\prime \prime}$ in the central $20^{\prime \prime}$. 


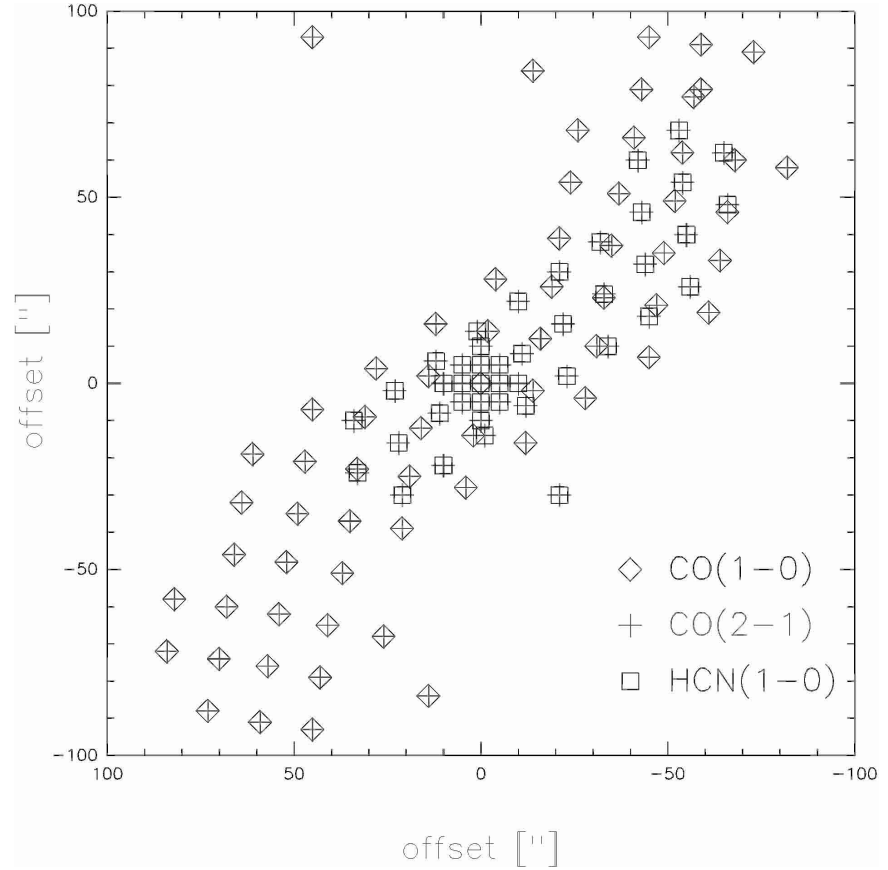

Fig. 3. A map of the pointing coordinates, observed at the two observing runs. The respective observed frequencies are marked as: black diamonds $(\mathrm{CO}(1-0))$, red crosses $(\mathrm{CO}(2-1))$ and blue squares $(\mathrm{HCN}(1-0))$.

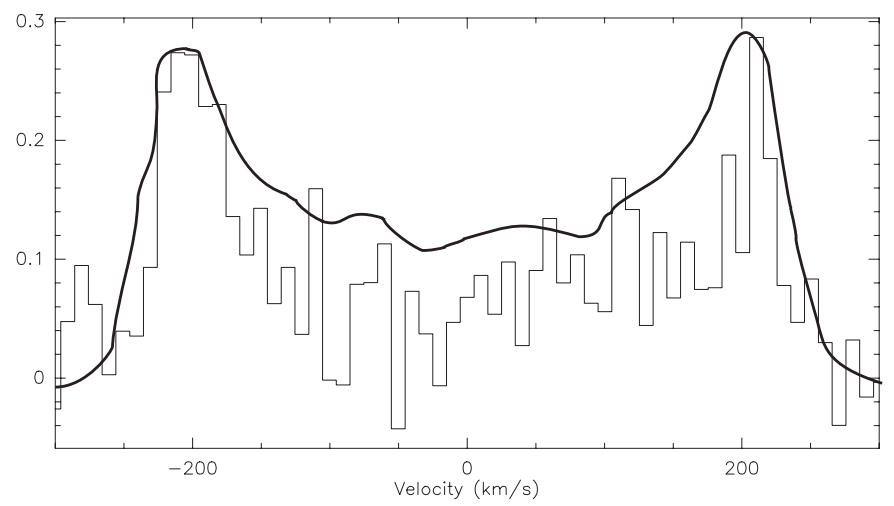

Fig. 4. Global $\mathrm{CO}(1-0)$ spectrum, obtained by summing up all individual spectra, measured at the positions given in Fig. 3. The intensity scale is in units of $T_{\mathrm{A}}^{*}(\mathrm{~K})$. To compare the global CO spectrum with the global HI spectrum, the latter is also shown as a solid line. The corresponding data are published in Schwarz (1985). The antenna temperature scale in the plot describes only the $\mathrm{CO}$ data.

\section{Observational results}

\subsection{Molecular gas distribution}

Maps of the integrated $\mathrm{CO}(1-0)$ and $\mathrm{CO}(2-1)$ line emission are shown in Figs. 5 and 6, respectively. The bulk of the emission is concentrated around the optical center of the galaxy (black cross). From this central location the distribution of the $\mathrm{CO}$ line emission shows in both transitions an extension towards the NW over a (deconvolved) length of $\sim 30^{\prime \prime}$, in agreement with the recently conducted high angular resolution observations using the IRAM Plateau de Bure interferometer (Krips et al., in prep.).

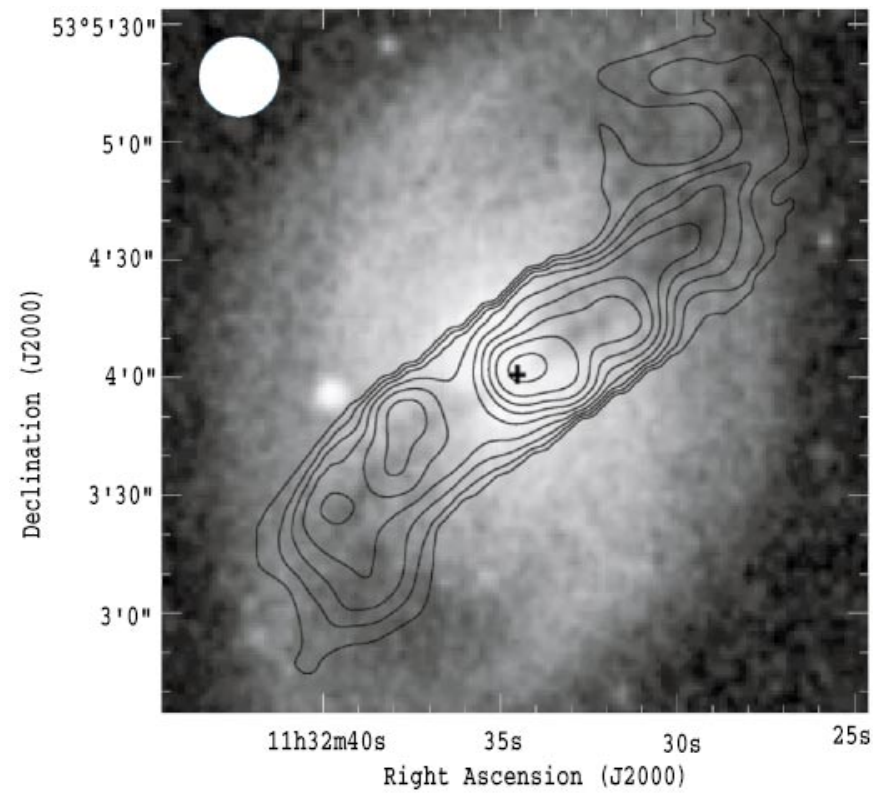

Fig. 5. Integrated line intensity map of $\mathrm{CO}(1-0)$ with linear contour lines (steps: $0.25 \mathrm{~K} \mathrm{~km} \mathrm{~s}^{-1}$ ). The observations were carried out in November 1999 at the IRAM $30 \mathrm{~m}$ telescope. The map is overlaid with an optical image of the galaxy, taken from the DSS survey. The cross indicates the optical center and the beamsize is presented in the upper left corner.

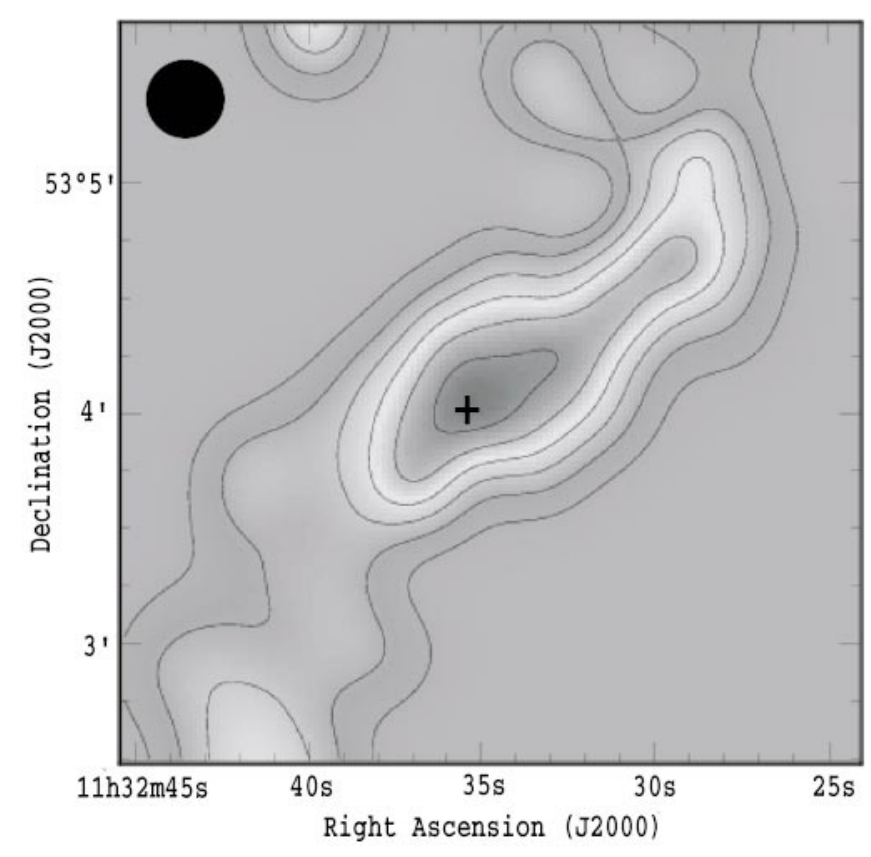

Fig. 6. Integrated intensity map of $\mathrm{CO}(2-1)$. The contours correspond to $0.2,0.4,0.6$ times $\mathrm{K} \mathrm{km} \mathrm{s}^{-1}$. The cross indicates the optical center. The map is convolved to the $21^{\prime \prime}$ resolution of the corresponding $\mathrm{CO}(1-0)$ observation, as indicated in the upper left corner.

A more extended component of the $\mathrm{CO}$ line follows the dust lane (see Fig. 5) along a PA of $114^{\circ}$. Deconvolved with the $115 \mathrm{GHz}$ and $230 \mathrm{GHz}$ beam sizes the width of this component perpendicular to the dust lane corresponds to about $35^{\prime \prime}$ $(\sim 2.2 \mathrm{kpc})$. As the modelling in Sect. 4 shows, this value is 


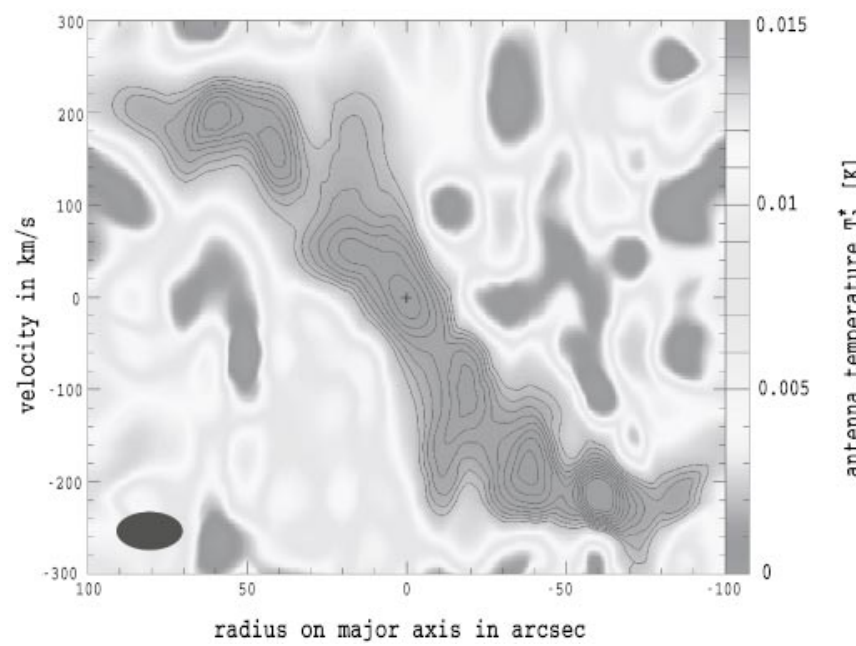

Fig. 7. The position-velocity-diagram of NGC 3718, obtained in $\mathrm{CO}_{1-0}$ along the major axis, with linear-scale contours. The cross indicates the optical center of the galaxy and the resolution is indicated by the pv-beam in the lower left corner.

dominated by the inclination and the warping of the molecular gas disk. The most striking result is the flaring of the distribution of the CO line emission at separations of about $R \sim 2.5 \mathrm{kpc}$ from the center (best visible in SE direction in Fig. 5). Roughly from here the line emission follows the HI warp of the disk that has been reported previously by Schwarz et al. (1985). At the $\mathrm{SE}$ tip of the disk, it is possible to follow the CO line emission at the $2 \sigma$ level up to $35^{\prime \prime}$ south of the dust lane, corresponding to $2.2 \mathrm{kpc}$ in projection. Both the atomic and the molecular gas share the same (projected) kinematics, suggesting that they are equally affected by the cause of the warp (see Fig. 4).

\subsection{Dynamical mass estimation}

If the gas in NGC 3718 is on near-circular orbits a rotation curve, i.e. a plot of the circular velocities, contains information about the enclosed mass as a function of separation from the nucleus. In the central region of the pv-diagram (Fig. 7) at $\sim 15^{\prime \prime}$ a velocity width of almost $200 \mathrm{~km} \mathrm{~s}^{-1}$ is observed. This indicates that the bulk of the molecular gas cannot be located in a simple rotating molecular gas disk. Therefore the derivation of a rotation curve is not straight forward. This feature is observed as well in HI by Verheijen \& Sancisi (2001) with a beam size of $12.9^{\prime \prime} \times 17.2^{\prime \prime}$ that falls between the angular resolutions obtained by the IRAM $30 \mathrm{~m}$ telescope in the $\mathrm{CO}(1-0)$ and $\mathrm{CO}(2-1)$ line.

In Sect. 4 we describe in detail how we derived an inclination corrected rotation curve $v(r)_{\text {corr }}$ from the HI and CO data that are available for NGC 3718. The enclosed mass can then be obtained via $m_{\text {circ }}(r)=v(r)_{\text {corr }}^{2} r G^{-1}$. Here $v(r)_{\text {corr }}=v(r)_{\text {obs }}$. $\sin ^{-1}(i)$ and $v(r)_{\text {obs }}$ is the observed rotation curve and $i$ the inclination. The quantities for the enclosed mass inside a radius of $90^{\prime \prime}$ are summarized in Table 3 up to $90^{\prime \prime}$, where the CO line emission is still significant (see Fig. 7). In Table 3 one can see the linear increase of the enclosed dynamical mass, induced by the constant rotation curve (Sect. 4.2). Within the central 10"
Table 3. Enclosed dynamical mass in the central region, based on the inclination-corrected rotation curve of our model in Sect. 4.

\begin{tabular}{ccc}
\hline \hline $\begin{array}{c}\text { Radius } \\
{[\operatorname{arcsec}]}\end{array}$ & $\begin{array}{c}v_{\text {corr }}(r) \\
{\left[\mathrm{km} \mathrm{s}^{-1}\right]}\end{array}$ & $\begin{array}{c}m_{\text {circ }} \\
{\left[10^{9} M_{\odot}\right]}\end{array}$ \\
\hline 10 & $235 \pm 15$ & 8.0 \\
20 & $235 \pm 15$ & 15.9 \\
30 & $235 \pm 15$ & 23.8 \\
60 & $235 \pm 15$ & 47.7 \\
90 & $235 \pm 15$ & 71.5 \\
\hline
\end{tabular}

corresponding to the $\mathrm{CO}$ beam-size, only $0.2 \%$ of the dynamical mass is contained in the molecular gas (cf. Sect. 3.3).

\section{3. $C O(2-1)$ to $C O(1-0)$ line ratio}

The ratio of the line intensities of $\mathrm{CO}(2-1)$ to $\mathrm{CO}(1-0)$ provides important information for the investigation of the physical conditions of the molecular gas. Therefore we present here the ratios resultant from our observations. A further discussion about obtaining and analysing molecular line ratios is given in Eckart et al. (1990a). Because the source extension is of the order of magnitude of the observing beam size, beam filling factors have been applied. Due to the observed integrated line intensity (Figs. 5 and 6) we assumed at the center an underlying Gaussian intensity profile of $\theta_{\mathrm{s}, \perp} \sim 35^{\prime \prime} F W H M$ orthogonal to the dust lane in Fig. 5. The extension along the dust lane appears to be much larger than the beam sizes. This lead to a correction about $\sim 10 \%$ in Eq. (1). Using the $\mathrm{CO}(1-0)$ and $\mathrm{CO}(2-1)$ spectra for the central position (cf. Figs. 8 and 9), we calculated the ratio ${ }^{2}$ :

$\frac{T_{21}^{\mathrm{mb}}}{T_{10}^{\mathrm{mb}}} \approx 0.84 \cdot f_{\text {cour. }}^{\text {source size }} \approx 0.75$.

The result in Eq. (1) is slightly smaller than the average found in 81 spiral galaxies by Braine et al. (1993) with a finding of $0.89 \pm 0.06$. We believe that the deviation of our result to the average value is rather reflecting beam size effects than intrinsic physical conditions (e.g. opacity) in the center of NGC 3718. Thus we assume that the intrinsic $T_{21}^{\mathrm{mb}} / T_{10}^{\mathrm{mb}}$-ratio of the core is close to unity, indicative of dense molecular gas. The beam size influences the measured central main beam temperature ratios, if already within the beam size the ratio decreases significantly. This decrease is indicated by the found mean line ratio of our observations at positions outside the central $20^{\prime \prime 3}$ :

$$
\frac{T_{21}^{\mathrm{mb}}}{T_{10}^{\mathrm{mb}}}=(0.46 \pm 0.14) .
$$

\footnotetext{
2 The antenna temperatures $T_{\mathrm{A}}^{*}$ are transformed into the main beam temperatures $T_{\mathrm{obs}}^{\mathrm{mb}}$ with the forward efficiency and the beam efficiency of the respective observation (see Table 2)

3 The central $20^{\prime \prime}$ are the area enclosed by a radius of $10^{\prime \prime}$.
} 


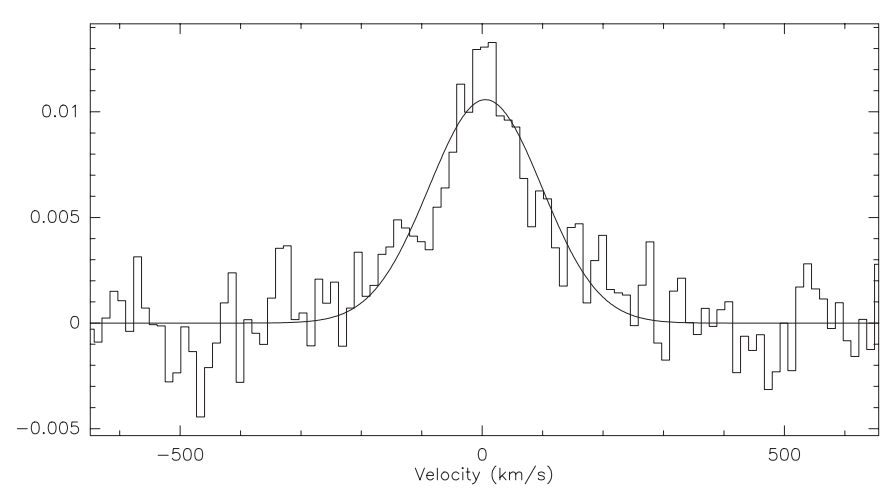

Fig. 8. The $\mathrm{CO}(1-0)$ spectrum in the center. Vertical scale is $T_{\mathrm{A}}^{*}(\mathrm{~K})$. The solid line shows a Gauss fit, with $I_{\mathrm{CO}(1-0)}=3.11 \mathrm{~K} \mathrm{~km} \mathrm{~s}^{-1}$.

From the central line intensity $I_{\mathrm{CO}_{10}}^{\mathrm{mb}}=4.45 \mathrm{~K} \mathrm{~km} \mathrm{~s}^{-1}$, which is corrected for the source size as discussed above, we estimated the central molecular gas mass ${ }^{4}$ for radii of $r \leq 10^{\prime \prime}$ :

$M_{\mathrm{H}_{2}}\left(r \leq 10^{\prime \prime}\right) \approx 2.1 \times 10^{7} M_{\odot}$.

From the total, source size corrected, line intensity, integrated over the sky:

$I_{\mathrm{CO}_{10}, \text { total }}^{\mathrm{mb}}=51.2 \mathrm{~K} \mathrm{~km} \mathrm{~s}^{-1} \mathrm{kpc}^{2}$

we calculated with the same conversion factor a total molecular gas mass of:

$M_{\mathrm{H}_{2}} \approx 1.8 \times 10^{8} M_{\odot}$.

To compare this value with other galaxies, we calculate the ratio to the dynamical mass, enclosed in the same region $\left(r \leq 120^{\prime \prime}\right)$, corresponding to the rotation velocity $v\left(120^{\prime \prime}\right)=$ $245 \mathrm{~km} \mathrm{~s}^{-1}$ (Fig. 13):

$M_{\text {dyn }}\left(r \leq 120^{\prime \prime}\right)=103.7 \times 10^{9} M_{\odot}$

$\rightarrow M_{\mathrm{H}_{2}} / M_{\mathrm{dyn}}\left(r \leq 120^{\prime \prime}\right)=0.17 \%$.

This value is low but still typical, compared with other nearby spiral galaxies (Sage 1993).

\subsection{FIR properties and the dust mass of NGC 3718}

Far-infrared continuum flux densities measured by IRAS (Table 4) allow us to characterize the dust in NGC 3718. The source is detected in all four bands. Following Lonsdale et al. (1985) we find a far-infrared luminosity of:

$L_{\mathrm{FIR}}=10^{8.45} L_{\odot}=2.8 \times 10^{8} L_{\odot}$.

Following (Wiklind et al. 1995) we could derive the dust temperature $^{5} T_{\text {dust }}$ and the dust mass $M_{\text {dust }}$ from the $60 \mu \mathrm{m}$ and $100 \mu \mathrm{m}$ IRAS flux densities:

$T_{\text {dust }}(60 \mu \mathrm{m}, 100 \mu \mathrm{m}) \approx 30 \mathrm{~K}$

\footnotetext{
${ }^{4}$ A conversion factor of

$\frac{N_{\mathrm{H}_{2}}}{I_{\mathrm{CO}_{10}}}=2.3 \times 10^{20}$ molecules $\mathrm{cm}^{-2}\left(\mathrm{~K} \mathrm{~km} \mathrm{~s}^{-1}\right)^{-1}$
}

(Strong et al. 1988) was used.

5 Assuming a mass opacity $\kappa_{v} \propto v$.

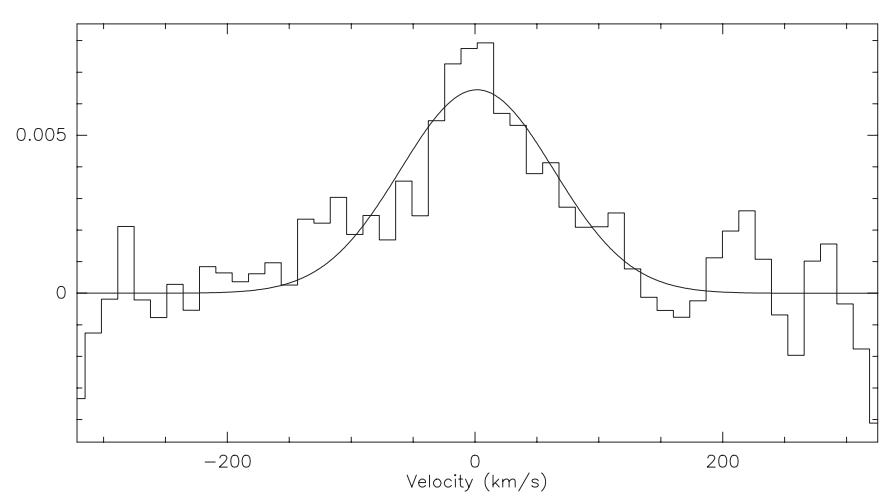

Fig. 9. The $\mathrm{CO}(2-1)$ spectrum in the center. Vertical scale is $T_{\mathrm{A}}^{*}(\mathrm{~K})$. The solid line shows a Gauss fit, with $I_{\mathrm{CO}(2-1)}=1.99 \mathrm{~K} \mathrm{~km} \mathrm{~s}^{-1}$.

Table 4. Flux data measured with IRAS.

\begin{tabular}{l|l}
\hline \hline \multicolumn{2}{c}{ IRAS fluxes } \\
\hline$f_{v}(12 \mu \mathrm{m})=0.169 \mathrm{Jy}$ & $f_{v}(60 \mu \mathrm{m})=0.697 \mathrm{Jy}$ \\
$f_{v}(25 \mu \mathrm{m})=0.097 \mathrm{Jy}$ & $f_{v}(100 \mu \mathrm{m})=2.29 \mathrm{Jy}$ \\
\hline
\end{tabular}

which is similar to NGC 5128 (Eckart et al. 1990b) and other galaxies (e.g. Wiklind et al. 1995; Leon et al. 1998). The estimated dust mass

$M_{\text {dust }}\left(T_{\text {dust }}^{60,100}, f_{v}^{100}\right) \approx 2.5 \times 10^{5} M_{\odot}$

leads to a gas-to-dust mass ratio of 720 in NGC 3718 which is close to the average typically observed value of the gas-to-dust ratio of about 700 (Wiklind et al. 1995; Leon et al. 1998), if the dust mass is derived from the $100 \mu \mathrm{m}$ flux density.

In the case of NGC 3718 it is therefore likely that a large amount of dust with temperatures of the order of $20 \mathrm{~K}$ or less is present and that the dust mass calculated above can only be a lower limit. Assuming a canonical gas-to-dust ratio of 100 we derive

$M_{\text {dust }}^{\text {theor. }} \sim 1.8 \times 10^{6} M_{\odot}$

from the molecular gas mass (Eq. (5)), which is likely to be much closer to the total dust mass of NGC 3718 .

Alton et al. (1998) showed that the $200 \mu \mathrm{m}$ measurements of the ISO satellite are more sensitive to colder dust components and noted that cold dust dominates at larger radii. Eckart et al. (1990b) gave a lower limit of $5.3 \times 10^{5} M_{\odot}$ for the dust content in NGC 5128, which is comparable to our estimation for NGC 3718 (Eq. (10)), and found a significant drop of the dust temperature towards the edges of the disk.

We adopt for NGC 3718 the same dust temperature drop due to the morphological and kinematical similarity between both galaxies (see Sect. 5). Thus the dust temperature of $30 \mathrm{~K}$ is valid for the center only.

The low temperatures, adopted for a large amount of dust in NGC 3718, are likely a consequence of a low interstellar radiation field heating the dust, and may particularly result from a very low massive star-formation rate (Devereux \& Hameed 1997). 


\subsection{Dense molecular gas and star formation}

We assume that the $\mathrm{HCN}$ emission in NGC 3718 is originating in dense gas from the center with temperature and kinematics similar to the emitting CO (cf. Sect. 3.3). Our HCN measurements towards NGC 3718 allow us to investigate the properties of the dense fraction of the molecular gas. While $\mathrm{CO}$ traces densities of $10^{2} \mathrm{~cm}^{-3} \leq n\left(\mathrm{H}_{2}\right) \leq 10^{4} \mathrm{~cm}^{-3}$, HCN traces dense molecular gas with $n\left(\mathrm{H}_{2}\right) \geq 10^{4} \mathrm{~cm}^{-3}$. Solomon et al. (1992) assumed that the emission lines trace gravitationally bound or virialized clouds. Using HCN radiative transfer solutions (e.g. Kwan \& Scoville 1975), Solomon et al. (1992) determined a molecular mass to $\mathrm{HCN}$ luminosity ratio for kinetic temperatures between 20 to $60 \mathrm{~K}$ of

$M_{\mathrm{HCN}}\left(\mathrm{H}_{2}\right) \approx 20_{-10}^{+30} \times L_{\mathrm{HCN}} M_{\odot}\left(\mathrm{K} \mathrm{km} \mathrm{s}^{-1} \mathrm{pc}^{2}\right)^{-1}$.

The HCN(1-0) line luminosities can be obtained from the integrated line intensities ${ }^{6} I_{\text {line }}=\int T^{\mathrm{mb}} \times \delta v$ via $L_{\text {line }}=I_{\text {line }} D^{2} \Omega$. $T^{\mathrm{mb}}$ is the main beam brightness temperature, $\mathrm{D}$ is the distance to the source and $\Omega$ is the solid angle of the beam convolved with the source. This gives an upper limit for the mass of dense gas of

$M_{\mathrm{HCN}}\left(\mathrm{H}_{2}\right) \leq 6.6 \times 10^{7} M_{\odot}$.

This value is of the same order but lower than the value of NGC 5128 of $M_{\mathrm{HCN}}\left(\mathrm{H}_{2}\right)=8.6 \times 10^{7} M_{\odot}$ (Wild \& Eckart 2000). As shown in Fig. 10 the found luminosity ratio of FIR to $\mathrm{HCN}(1-0)$ line emission in NGC 3718 is similar to the corresponding ratios of ULIRGs and normal spirals.

The luminosity ratio between $\mathrm{CO}(1-0)$ and $\mathrm{HCN}(1-0)$ emission over the $\mathrm{CO}$ mapped region is $\mathfrak{R}_{\mathrm{CO} / \mathrm{HCN}} \geq 15.5$. Curran et al. (2001) found in a recent survey of Seyfert galaxies similar values. A low fraction of dense gas and thus a low star formation activity in NGC 3718 can be responsible for the fact that we can only estimate an upper limit for the HCN luminosity.

The HCN emission in external galaxies is usually stronger peaked on the nucleus compared to the $\mathrm{CO}$ emission. In NGC 3718 at the central position, the integrated intensity ratio $I(\mathrm{HCN}(1-0)) / I(\mathrm{CO}(1-0))$ is less than 0.05 . Ratios of the order of 0.1 or higher are only observed in active nuclear regions of Seyfert galaxies and ULIRGs (Kohno et al. 1996; Solomon et al. 1992; Nguyen et al. 1992; Helfer \& Blitz 1993, 1995; Jackson et al. 1993; Tacconi et al. 1994). Sternberg et al. (1994) found large $\mathrm{HCN} / \mathrm{CO}$ intensity ratios in the nucleus of the nearby Seyfert 2 NGC 1068 and argued, that, in part, high ratios can also result from a selective depletion of gas-phase oxygen.

Wild \& Eckart (2000) found in the center of Cen A an integrated intensity ratio $I(\mathrm{HCN}(1-0)) / I(\mathrm{CO}(1-0)) \leq 0.064$ which is comparable to the upper limit given above, but falls well below the value for ULIRGs. The ratio of $\mathrm{HCN}$ to CO luminosity is $1 / 6$ for ULIRGs, but only $1 / 80$ in normal spirals (Solomon et al. 1992).

${ }^{6}$ Which represent the beam-diluted brightness temperatures of the source.

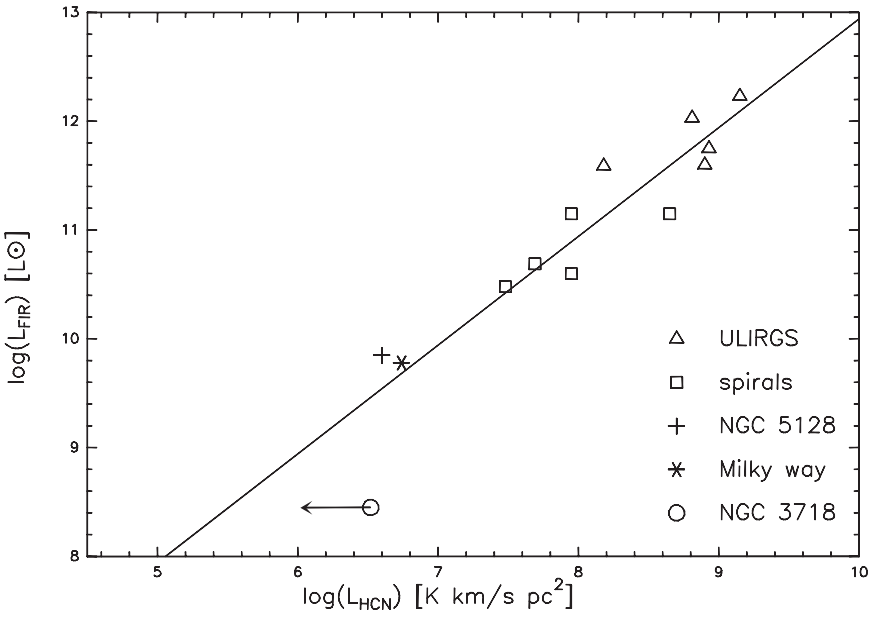

Fig. 10. NGC 3718 has the same ratio of FIR to HCN luminosity as ULIRGs (black triangles) and normal spirals (red squares). NGC 5128 (blue asterisk) and the Milky Way (green cross) are shown for comparison as well. The blue circle shows the upper limit of $L_{\mathrm{HCN}}$ for NGC 3718. All data except our own are taken from Solomon et al. (1992).

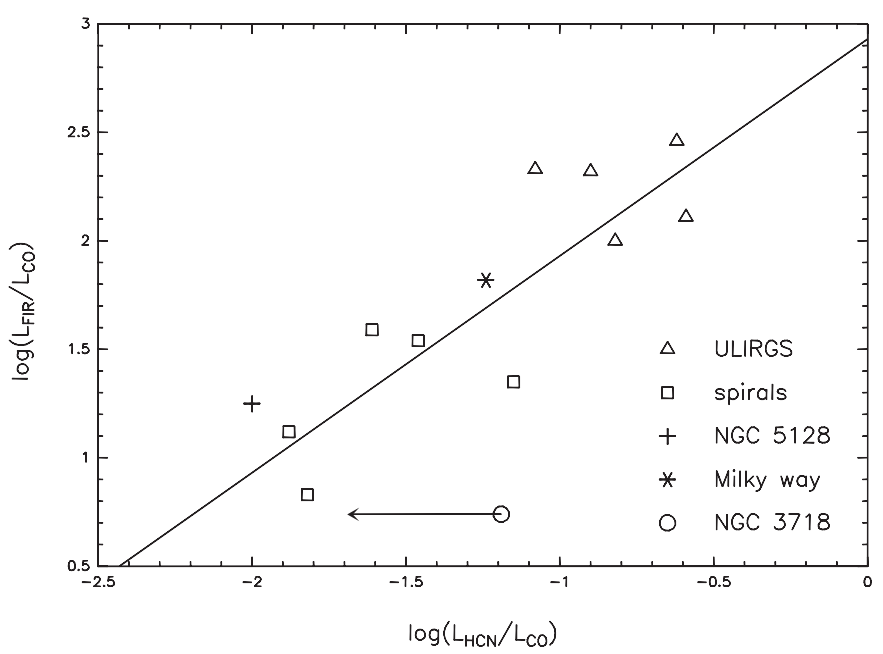

Fig. 11. Comparison of the fraction of dense gas to the star formation efficiency (Solomon et al. 1992). Symbols are the same as in Fig. 10.

Figure 10 shows that for NGC 3718 both the FIR and the HCN luminosity fall even below that of normal spirals. This suggests that the presence of dense gas and dust heated by young stars is less pronounced in NGC 3718 compared to spirals. It also suggests that the star formation rate per mass of dense gas is smaller.

In Fig. 11 we show the position of NGC 3718 in a plot of $\log \left(L_{\mathrm{HCN}} / L_{\mathrm{CO}}\right)$ which measures the fraction of dense gas versus $\log \left(L_{\mathrm{FIR}} / L_{\mathrm{CO}}\right)$. The latter ratio measures the efficiency with which molecular gas is transformed into OB stars (Solomon et al. 1992). Also this plot includes ULIRGs as well as galaxies of normal infrared luminosity. While the fraction of dense gas may be similar (or below) to values obtained for normal galaxies, we find that the star formation efficiency is clearly at the lower end of the range of values covered by normal galaxies. 


\section{Results of the kinematic modelling}

\subsection{The tilted-ring model}

The high degree of symmetry of our observations of the molecular gas both in the integrated CO line intensity distribution (Fig. 5) and in the pv-diagram (Fig. 7) along the galaxy's major axis motivated us to create a tilted-ring model of the gas distribution. Its basic idea is to settle the gas in concentric rings around the galactic nucleus. The particles are circling on these orbits with the velocity, given by the corresponding rotation curve. Thus non-circular motion is neglected. If all orbits would be located and oriented in one plane, the gas distribution would equal a rotating disk. The crucial point of the modelling process is the variation of the location of the single orbits and of the rotation curve. At one radius only one orbit-location with one rotation velocity is allowed. Usually the innermost molecular gas is located in a disc, i.e. the rings are located in the same plane. With increasing radius the outer gas rings are inclined against this central disc. Inclined orbits are precessing with respect to the central plane with a precession-velocity proportional to the rotation velocity of the single particles.

The fitting process can be described in two steps. The orbit orientation and the rotation velocities are varied under the assumption of a continuous variation with increasing radius. Then artificial maps are calculated and compared with the real ones. These steps are repeated in an iterative manner until a satisfying parameterset is found. Details on the history of these models and the used software are described by Schinnerer et al. (2000a). Tilted-ring models have been applied successfully to several galaxies, pioneering work was done by Rogstad et al. (1974, 1976), who modelled the HI distribution in the galaxies M 83 and M 33.

\subsection{Derivation of the input parameters}

For the tilted-ring model we need several input parameters:

\section{rotation curve:}

From our observations we could derive the inner rotation curve $v\left(r \leq 100^{\prime \prime}\right)$ by analyzing the pv-diagram (Fig. 7), which is calculated along the major axis of the integrated $\mathrm{CO}$-line intensity. Because orbit crowding occurs along this axis, Fig. 7 does not show the usual simple pv-shape of a single rotating disk. For example in the inner $20^{\prime \prime}$ one observes in Fig. 7 a steep linear feature similar to a fast rotating disk of $r \sim 20^{\prime \prime}$. Additionally the central peak is elongated into another direction. Further secondary maxima occur at $r \sim \pm 20^{\prime \prime}$. At least a significant partition of the central maximum is contributed by the outer gas rings $\left(r \geq 90^{\prime \prime}\right)$, which are seen nearly edge-on. Therefore they are also populating the inner region (i.e. close to the centre and velocities close to the systemic one) in the pv-diagram. The above mentioned secondary maxima are contributed by inclined orbits at intermediate radii. In general the intrinsic orbital velocity at a given radius $r_{0}$ is correlated to the highest velocities, detected at this distance to the center. But the maximum observed velocity at $r_{0}$ has still to be corrected by the inclination of the corresponding orbit to give the

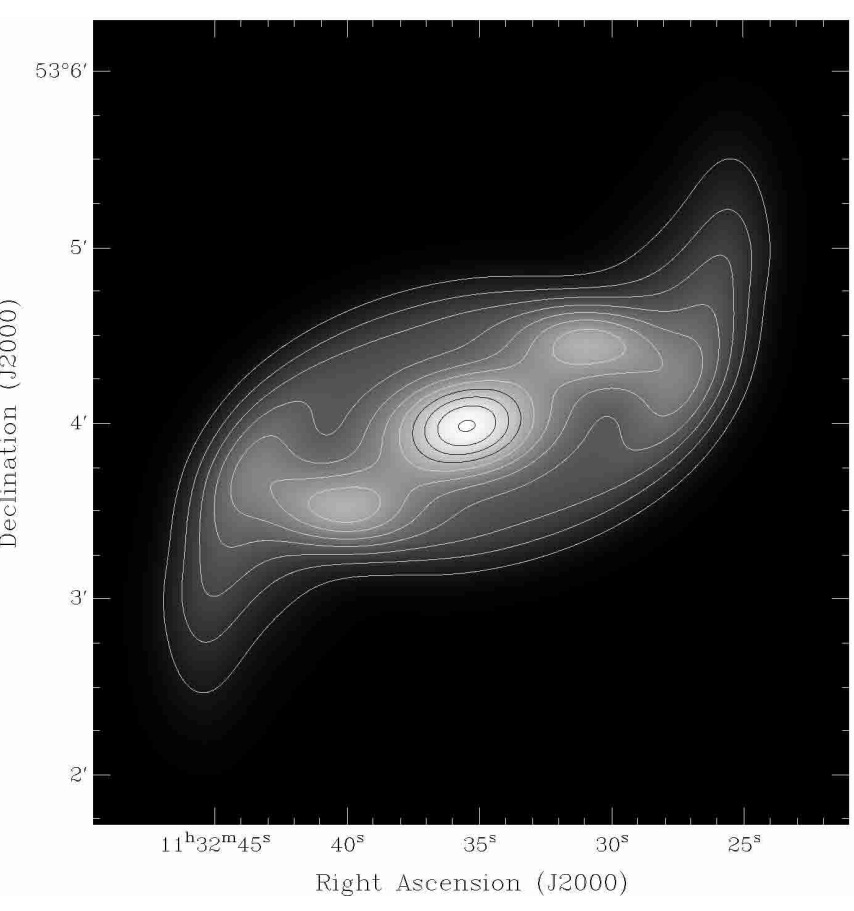

Fig. 12. The modeled integrated $\mathrm{CO}(1-0)$ line intensity shown with linear-scale contours.

intrinsic velocity $v\left(r_{0}\right)$. The uncertainties of the derived intrinsic velocities cannot be alleviated below $10-15 \mathrm{~km} \mathrm{~s}^{-1}$ due to these dependances.

A careful analysis of (Fig. 7) reveals, that some features of the north-western region (right part in Fig. 7) occur at velocities slightly differing from their south-eastern counterparts. In these cases we chose mean values for the symmetric model.

The final fit confirmed a flat rotation curve of $v\left(10^{\prime \prime} \leq\right.$ $\left.r \leq 100^{\prime \prime}\right)=235 \mathrm{~km} \mathrm{~s}^{-1}$ within the errors. The shape of the fitted rotation curve (Fig. 13) is a quite typical one (cf. Sofue 1996). In the transition region between our data and the HI-data of Schwarz (1985), we adopted a linear increase to the nearly constant velocities $\left(v_{\mathrm{HI}}\left(r \geq 100^{\prime \prime}\right) \approx\right.$ $255 \mathrm{~km} \mathrm{~s}^{-1}$ ) of the atomic gas. No evidence was found for extraordinary velocities at these radii, which would reject such an adoption.

The apparent excess of high velocities $\left(\sim 200 \mathrm{~km} \mathrm{~s}^{-1}\right)$ near the center of the galaxy $\left(\sim 20^{\prime \prime}\right)$ in Fig. 7 demonstrates the existance of high intrinsic velocities close to the center. This important feature could not be resolved by the lower resolution HI data of Schwarz (1985) but is consistent with the central ( $\left.\leq 80^{\prime \prime}\right) \mathrm{H}_{\alpha}$ data of Reshetnikov $\&$ Combes (1994). This excess, reproduced by our model (Fig. 14), is not found as a peak in the intrinsic rotation curve (Fig. 13). The modelfit shows convincingly, that the apparent decrease of high velocities at $r \geq 20^{\prime \prime}$ is only observed due to the different inclination of the respective orbits.

galactic disc:

The orientation of the galactic disc in space is fixed with an inclination of $i=(60 \pm 10)^{\circ}$ and a position angle (PA) of 


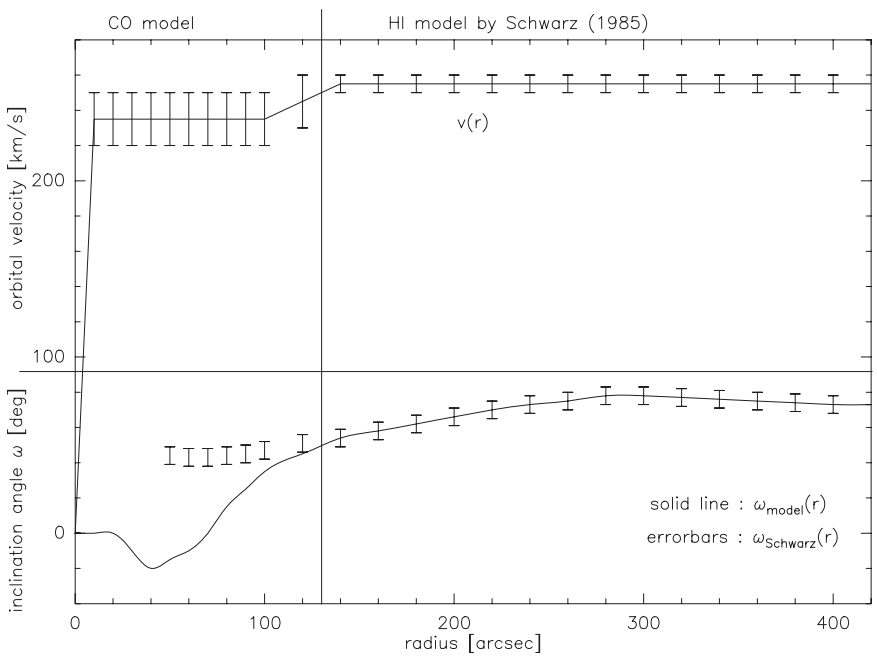

Fig. 13. The upper panel shows the rotation curve $v(r)$ used to model NGC 3718, in the lower panel the corresponding tilting-angles $\omega(r)$ are plotted.

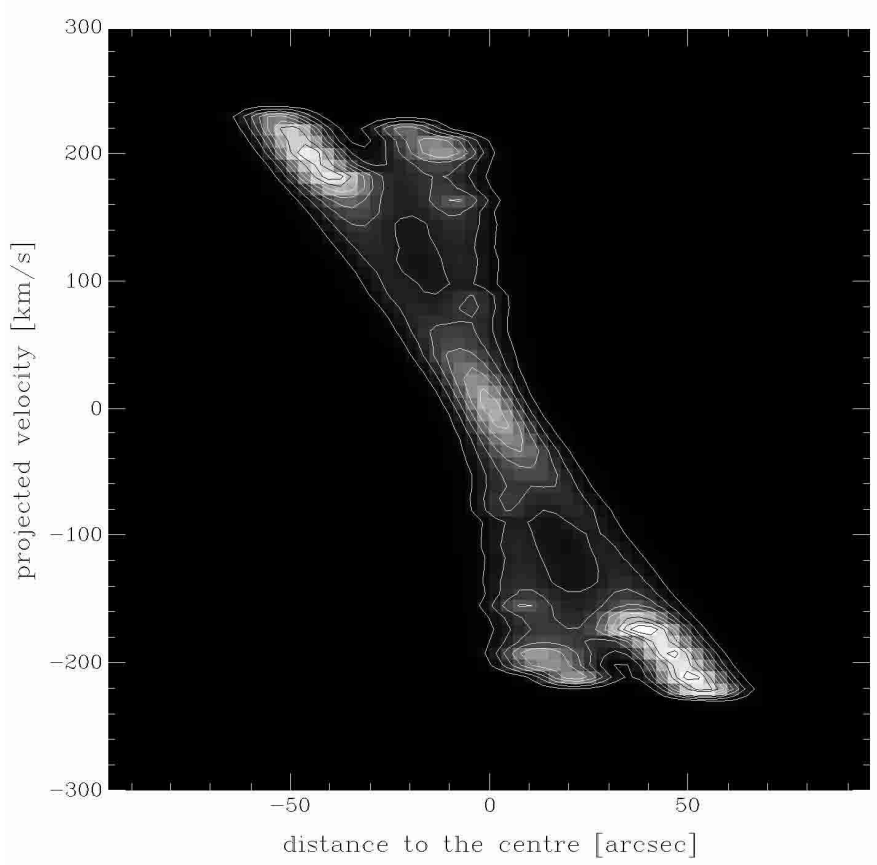

Fig. 14. The modeled pv-diagram along the major axis of the integrated line-intensity. A linear contour-scale is shown.

$(-85 \pm 5)^{\circ}$. It assigns the frame of reference, in which the parameters curve of nodes and tilt angles are defined (see next section). The position angle can be deduced from the PA of the overall emission in the integrated line intensity map. The inclination is deduced from the ellipticity of the mapped integrated line emission assuming an overall circular shape of the molecular gas disk. During the iterative model fit also the frame of reference is varied.

curve of nodes $\mathcal{E}$ tilt angles:

Thirdly the spatial orientation of the gas rings has to be determined. It is represented by the curve of nodes $\alpha(r)$ and the tilt angles $\omega(r)$. The former defines for every given

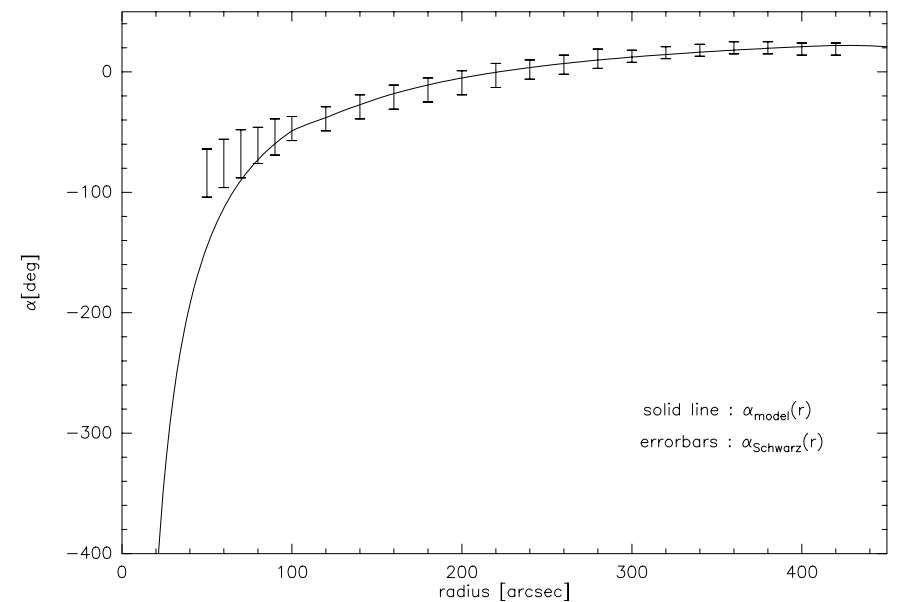

Fig. 15. The plot shows the curve of nodes of our model (solid line), discussed in Sect. 4. In our model the warp starts at $20^{\prime \prime}$, as one can see in Fig. 13. Furthermore the errorbars are indicating the corresponding values of the HI-rings (Schwarz 1985) in the same frame of reference.

radius the line of intersection between the galactic plane and the plane of the tilted ring (Schinnerer et al. 2000a). The latter gives the angle between these two planes. To find the right combination of $\alpha(r)$ and $\omega(r)$, we assumed a continuous, smooth variation of the tilt angles (starting with $\omega\left(r \leq 20^{\prime \prime}\right)=0$ for the central disc). Furthermore the general shape of $\alpha(r)$ is determined by the rotation curve as described by Schinnerer et al. (2000a):

$\alpha(r)=\epsilon \Delta t \frac{v(r)}{r}+\alpha_{0}$.

The last equation is derived from the fact, that in other than spherically symmetric potentials the curve of nodes evolves with time (i.e. the rings are precessing). Thus $\alpha(r)$ can reveal in combination with the tilt angles of the rings information about the gravitational potential of the galaxy and the causes of the found warp.

The above considerations lead to a physically reasonable set of input parameters for the tilted-ring model, which is consistent both with our high resolution data of the molecular gas and the HI-data, whose spatial resolution was recently improved by Verheijen \& Sancisi (2001) with respect to the observations of Schwarz (1985).

\subsection{Results}

Under the boundary conditions described above we varied $\omega$, $\epsilon \Delta t$ and $\alpha_{0}$, plotted integrated line-intensity maps and pvdiagrams of our model and compared them with Figs. 5 and 7. The resulting best fit parameter curves are presented in Figs. 13 and 15 , error estimates of $\left(\Delta \alpha= \pm 10^{\circ}\right)$ and $\left(\Delta \omega= \pm 5^{\circ}\right)$ are characterizing the accuracy of the fits. As shown in Fig. 15, we could reveal a smooth transition to the curve of nodes of Schwarz (1985), who developed a tilted-ring model for the HI distribution.

Figures 12 and 14 demonstrate that our model provides an excellent explanation for the observed data of the 
Table 5. Properties of NGC 3718 and NGC 5128.

\begin{tabular}{|c|c|c|c|}
\hline & NGC 3718 & NGC 5128 & references \\
\hline Host type & $\mathrm{Sa}^{a}$ & S0pec ${ }^{b}$ & ${ }^{a}$ Verheijen \& Sancisi $(2001) ;{ }^{b}$ NED \\
\hline Distance; Linear scale $\left[\frac{\mathrm{pc}}{\operatorname{arcsec}}\right]$ & $13.2 \mathrm{Mpc} ; 64^{b}$ & $3.4 \mathrm{Mpc} ; 16.5^{c}$ & ${ }^{b} \mathrm{NED} ;{ }^{c}$ Israel (1998) \\
\hline App. $B$-magnitude & $11.59 \mathrm{mag}$ & $7.84 \mathrm{mag}$ & ${ }^{b} \mathrm{NED}$ \\
\hline Total mass & $4.4 \times 10^{11} M_{\odot}^{d}$ & $(3 \pm 1) \times 10^{11} M_{\odot}^{e}$ & ${ }^{d}$ Dickel \& Rood (1978); ${ }^{e}$ Mathieu et al. (1996) \\
\hline Molecular gas mass $\left(M_{\odot} 10^{8}\right)$ & 1.8 & $2^{f}$ & ${ }^{f}$ Wild \& Eckart (2000) \\
\hline Dust mass $\left(M_{\odot} 10^{5}\right)$ & 2.5 & $5.3^{g}$ & ${ }^{g}$ Eckart et al. (1990b) \\
\hline$M_{\mathrm{HCN}}\left(\mathrm{H}_{2}\right)\left(10^{7} M_{\odot}\right)$ & $\leq 6.6$ & $8.6^{f}$ & ${ }^{f}$ Wild \& Eckart (2000) \\
\hline$L_{\mathrm{CO}}\left(10^{6} \mathrm{~K} \mathrm{~km} \mathrm{~s}^{-1} \mathrm{pc}^{2}\right)$ & 51.2 & $92^{f}$ & ${ }^{f}$ Wild \& Eckart (2000) \\
\hline$L_{\mathrm{HCN}}\left(10^{6} \mathrm{~K} \mathrm{~km} \mathrm{~s}^{-1} \mathrm{pc}^{2}\right)$ & $\leq 3.3$ & $5.5^{f}$ & ${ }^{f}$ Wild \& Eckart (2000) \\
\hline$L_{\mathrm{FIR}}\left(10^{9} L_{\odot}\right)$ & 0.28 & $6.0^{f}$ & ${ }^{f}$ Wild \& Eckart (2000) \\
\hline $\mathrm{S}(8.4 \mathrm{GHz})$ & $6.6 \mathrm{mJy}^{h}$ & $1.74 \mathrm{Jy}^{i}$ & ${ }^{h}$ Nagar et al. (2001), ${ }^{i}$ Wright \& Otrupcek (1990) \\
\hline Position angle of major axis ${ }^{k}$ & $-85^{\circ}$ & $125^{\circ l}$ & ${ }^{l}$ Sparke $(1996)$ \\
\hline Inclination $^{k}$ & $60^{\circ}$ & $73^{\circ l}$ & ${ }^{l}$ Sparke (1996) \\
\hline
\end{tabular}

${ }^{b}$ NED: NASA/IPAC Extragalactic Database.

${ }^{k}$ For a prominent ring or disc in the molecular gas, which can be usedas the frame of reference for a tilted ring-model; see Sect. 4.

molecular gas, both in the integrated line intensities (Fig. 5) and the pv-diagram (Fig. 7). Observed features (e.g. the strong warp, the secondary maxima in the integrated $\mathrm{CO}$-line intensity at $\sim 45^{\prime \prime}$ distance to the central peak (Fig. 5) and the large velocity dispersion at $\sim 20^{\prime \prime}$ (Fig. 7)) are shown to be the result of orbit crowding. No hotspots, isolated GMCs and other features have to be added to a standard gas distribution to describe the observed secondary maxima. Only a smooth radius-dependent variation of the inclination of the orbits is needed.

Also the high degree of symmetry found a natural explanation. We are able to confirm that in the transition region between the outer atomic gas distribution and the inner molecular gas around $r \sim 100^{\prime \prime}$ both fractions of the neutral gas are located in the same orbits with the same velocities. And the rotation curve of the molecular gas could be estimated down to a radius of $r=10^{\prime \prime}$ by deprojecting the data via the iterative fitting process.

\section{Comparison with NGC 5128}

NGC 5128 is the lenticular galaxy hosting the famous radio source Centaurus A. A comparison of its general properties with those of NGC 3718 is given in Table 5. A review of the current knowledge about NGC 5128 is given by Israel (1998). As outlined in the following especially the molecular gas disks of both objects are very similar to each other.

In Fig. 2 (plate 9) in Eckart et al. (1990b), the superposition is shown of integrated $\mathrm{CO}(1-0)$ line intensity map with the optical map of NGC 5128. This plate is equivalent to Fig. 5. Again a prominent dust lane covering the nucleus indicates a highly inclined gas disk. But more detailed kinematical analysis revealed that the molecular gas distribution can be described much better by a tilted-ring model than with a simple rotating gas disk (see Figs. 8d and e in Quillen et al. 1992).

The data and models presented by Eckart et al. (1990b), Quillen et al. (1992, 1993), and van Gorkom et al. (1990) give a strong support to their tilted-ring model for the molecular gas distribution of NGC 5128. They definitely show that the molecular gas distribution also agrees with the distribution of dust and atomic gas at larger radii, similar to NGC 3718.

The data in Table 5 indicate that the total mass estimates and the molecular gas masses of both galaxies are comparable. Using the tilted-ring models it is possible to compare also the intrinsic kinematical data by deprojection of the observations as described in Sect. 4. Quillen et al. (1992) estimated a shape of the rotation curve of NGC 5128 which clearly resembles Fig. 13: a linear increase at radii smaller than $40^{\prime \prime}$ which corresponds to $10^{\prime \prime}$ in NGC 3718 due to the different distance. For radii beyond the stellar bulge the rotation curve approaches asymptotically a constant value of $290 \mathrm{~km} \mathrm{~s}^{-1}$, slightly higher than the rotation velocity of the molecular gas in NGC 3718. Due to the comparable molecular gas content this indicates a higher inner dynamical mass in NGC 5128. Summarizing we state here that the ISM in both galaxies show very similar intrinsic kinematical properties, exceeding the simple agreement of the observed integrated intensity maps. This suggests that also the formation history of the observed warped gas disks may be comparable.

In contrast to the equal molecular gas content the FIR luminosity of NGC 3718 is substantially smaller than the one of NGC 5128. The nuclear FIR luminosity of NGC 5128 is weak compared with the luminosity originating from the extended star forming molecular gas disk (Wild \& Eckart 2000). Thus the smaller $L_{\mathrm{FIR}}$ in NGC 3718 may also indicate a lower star formation activity compared to NGC 5128.

The appearance of NGC 5128 (Cen A) in the radio regime differs significantly from NGC 3718. It is classified as Fanaroff-Riley class I (Israel 1998). The radio luminosity (Table 5) of both galaxies differ by about roughly one order of magnitude. Furthermore in NGC 3718 no extended radio continuum structure (e.g. lobes, jets) has been found apart from the central core. This implies intrinsic differences in the 
processes radiating at $\mathrm{cm}$-wavelengths. But especially in case of NGC 3718, more observational information is needed to support such a statement.

The morphological analogy of both warped gas disks even holds for the gas kinematics. This suggests, that probable causes for strong warped gas disks, such as past interaction and/or merger events, might not have a strong influence on the radio continuum properties of a galaxy, which are shown to be quite different in both galaxies. A similar conclusion could be drawn for the star formation activity. The lower star formation activity in NGC 3718 , as suggested by the lower FIR luminosity, may be uncorrelated to strong warps and their creation history.

\section{Summary}

Our three-dimensional model explains convincingly the observed molecular gas distribution of NGC 3718 in all details of the underlying data. The total gas kinematics are traced down to the limiting resolution of our single-dish measurements. The employed tilted ring model could be succesfully expanded to the outer atomic gas distribution, too. Thus it also explains the earlier observations of HI by Verheijen \& Sancisi (2001) and Schwarz (1985), obtained at different spatial resolutions.

Our model clearly shows, that the warp of the neutral gas distribution continues down to a radius of $20^{\prime \prime}$. This is represented by the tilt angles, which are unequal to zero for $r \geq 20^{\prime \prime}$ (Fig. 13). The data of the HI-observations could not lead to a comparable model at such small radii due to their lower spatial resolution and the depletion of atomic gas in the central region. Furthermore by fitting the line intensity of the modeled gas orbits to the observed integrated line intensity map, we found only for small radii within the central $10^{\prime \prime}$ a significant rise of the (intrinsic) intensity. Outside a constant or slightly decreasing line intensity with increasing orbit radius was fitted. This result could not be derived directly from the integrated line-intensity map without creating a complete model for the molecular gas kinematics.

The successful application of the tilted-ring model corroborates the assumption, that the neutral gas of NGC 3718 is mainly distributed in concentric orbits. Similar to NGC 5128 (Quillen et al. 1993) the kinematical behavior of the molecular gas of NGC 3718 can be totally described by a precessing warped gas disk. A molecular bar and non-circular motion of the gas was not found down to the central $500 \mathrm{pc}$.

We hope that our model can be employed to analyse the galactic gravitational potential in NGC 3718 and to find hints to its formation conditions and to the causes of the observed strong warp. The comparison with NGC 5128 suggests, that a strong warp in the neutral gas distributions down to 500 pc may be uncorrelated to the radio continuum properties of the galaxy.

Acknowledgements. We thank the staff at IRAM for their assistance and hospitality during the observations. This work was supported in part by the Deutsche Forschungsgemeinschaft (DFG) via grant SFB 494, by special funding from the Science Ministry of the Land Nordrhein-Westfalen. S. Leon is supported by a Marie Curie Individual Fellowship contract HPMF-CT-20002-01734 from the European Union.

\section{References}

Arp, H. 1966, Atlas of Peculiar Galaxies (Pasadena, CA: California Institute of Technology)

Arp, H. 1973, ApJ, 185, 797

Alton, P. B., Trewhella, M., Davies, J. I., et al. 1998, A\&A, 335, 807

Barth, A. J., Ho, L. C., Filipenko, A. V., \& Sargent, W. L. W. 1998, ApJ, 496, 133

Braine, J., Combes, F., Casoli, F., et al. 1993, A\&AS, 97, 887B

Burke, B. F., \& Miley, G. K. 1973, A\&A, 28, 379

Combes, F., Debbasch, F., Friedli, D., \& Pfenniger, D. 1990, A\&A, 233,82

Condon, J. J. 1987, ApJS, 65, 485

Curran, S. J., Polatidis, A. G., Aalto, S., et al. 2001, A\&A, 373, 459

Devereux, N. A., \& Hameed, S. 1997, AJ, 113, 599

Dickel, J. R., \& Rood, H. J. 1978, ApJ, 223, 391

Dufour, R. J., Harvel, C. A., Martins, D. M., et al. 1979, AJ, 84, 284

Duschl, W. J., Strittmatter, P. A., \& Biermann, P. L. 2000, A\&A, 357, 1123

Eckart, A., Downes, D., Genzel, R., et al. 1990a, ApJ, 348, 434

Eckart, A., Cameron, M., Rothermel, H., et al. 1990b, ApJ, 363, 451

Eckart, A., Cameron, M., Jackson, J. M., et al. 1991, ApJ, 372, 67

Eckart, A., \& Downes, D. 2001, ApJ, 551, 730

Englmaier, P., \& Shlosman, I. 2000, ApJ, 528, 677

Filippenko, A. V., \& Sargent, W. L. W. 1985, ApJS, 57, 503

Garcia, A. M. 1993, A\&AS, 100, 47

García-Burillo, S., Sempere, M. J., Combes, F., Hunt, L. K., \& Neri, R. 2000, A\&A, 363, 869

García-Burillo, S., Combes, F., Eckart, A., et al. 2003a, Active Galactic Nuclei: from Central Engine to Host Galaxy, meeting held in Meudon, France, July 23-27, 2002, ed. S. Collin, F. Combes, \& I. Shlosman., ASP (Astronomical Society of the Pacific), Conf. Ser., 290, 423

García-Burillo, S., Combes, F., Hunt, L. K., et al. 2003b, A\&A, in press

Greenhill, L. J., \& Gwinn, C. R. 1997, Ap\&SS, 248, 261

Helfer, T. T., \& Blitz, L. 1993, BAAS, 25, 916

Helfer, T. T., \& Blitz, L. 1995, ApJ, 450, 90

Ho, L. C., Filippenko, A. V., \& Sargent, W. L. W. 1997, ApJS, 112, 315

Holmberg, E. 1958, Meddelanden fran Lunds Astronomiska Obersvatorium Seri II, 136, 1

Hummel, E. 1980, A\&AS, 41, 151

Israel, F. P. 1998, A\&ARv, 8, 237

Jackson, J. M., Heyer, M. H., \& Paglione, T. A. D. 1993, BAAS, 25 , 1467

Kohno, K., Kawabe, R., Tosaki, T., \& Okumura, S. K. 1996, ApJ, 461, L29

Kwan, J., \& Scoville, N. 1975, ApJ, 195, L85

Leon, S. Combes, F., \& Menon, T. K. 1998, A\&A, 330, 37

Lonsdale, C. H., Helou, G., Good, J. C., \& Rice, W. 1985, Cataloged Galaxies and Quasars Observed in the IRAS Survey, NASA-JPL, Pasadena

Maoz, D., Filippenko, A. V., Ho, L. C., et al. 1995, ApJ, 440, 91

Mathieu, A., Dejonghe, H., \& Hui, X. 1996, A\&A, 309, 30

Miyoshi, M., Herrnstein, J., Greenhill, L., et al. 1995, Nature, 373, 127

Nagar, N. M., Wilson, A. S., \& Falcke, H. 2001, ApJ, 559, L87

Nguyen, Q.-R., Jackson, J. M., Henkel, C., Truong, B., \& Mauersberger, R. 1992, ApJ, 399, 521

Niklas, S., Klein, U., Braine, J., et al. 1995, A\&AS, 114, 21

Pierce, M. J., \& Tully, R. B. 1988, PASP, 100, 1224

Quillen, A. C., de Zeeuw, P. T., Phinne, E. S., \& Phillips, T. G. 1992, ApJ, 391, 121 
Quillen, A. C., Graham, J. R., \& Frogel, J. A. 1993, ApJ, 412, 550

Reshetnikov, V. P., \& Combes, F. 1994, A\&A, 291, 57

Rogstad, D. H., Lockart, I. A., \& Wright, M. C. H. 1974, ApJ, 193, 309

Rogstad, D. H., Wright, M. C. H., \& Lockhart, I. A. 1976, ApJ, 204, 703

Sage, L. J. 1993, A\&A, 272, 123

Sandage, A. 1961, The Hubble atlas of galaxies (Washington: Carnegie Institution, 1961)

Schinnerer, E., Eckart, A., \& Tacconi, L. J. 2000a, ApJ, 533, 826

Schinnerer, E., Eckart, A., Tacconi, L. J., et al. 2000b, ApJ, 533, 850

Schwarz, U. J. 1985, A\&A, 142, 273

Shlosman, I., Frank, J., \& Begelman, M. C. 1989, Nature, 338, 45

Sparke, L. S. 1996, ApJ, 473, 810

Sofue, Y. 1996, ApJ, 458, 120

Solomon, P. M., Downes, D., \& Radford, S. J. E. 1992, ApJ, 387, L55
Sternberg, A., Genzel, R., \& Tacconi, L. 1994, ApJ, 436, L131

Strong, A. W., Bloemen, J., Dame, T., et al. 1988, A\&A, 207, 1

Tacconi, L. J., Genzel, R., Blietz, M., et al. 1994, ApJ, 426, L77

Tully, R. B., Verheijen, M. A. W., Pierce, et al. 1996, AJ, 112, 2471

van Gorkom, J. H., van der Hulst, J. M., Haschick, A. D., \& Tubbs, A. D. 1990, AJ, 99, 1781

Verheijen, M. A. W., \& Sancisi, R. 2001, 370, 765

de Vaucouleurs, G., de Vaucouleurs, A., Corwin, H. G., et al. 1991, Third Reference Catalogue of Bright Galaxies, Vol. 1-3, XII, 2069, pp. 7 figs (Berlin Heidelberg, New York: Springer-Verlag)

Wiklind, T., Combes, F., \& Henkel, C. 1995, A\&A, 297, 643

Wild, W., Eckart, A., \& Wiklind, T. 1997, A\&A, 322, 419

Wild, W., \& Eckart, A. 2000, A\&A, 359, 483

Wright, A., \& Otrupcek, R. 1990, Parkes Catalog, Australia telescope national facility 